\title{
Radiation-induced hepatitis B virus reactivation in hepatocellular carcinoma: A case report
}

\author{
JUN CHENG $^{1^{*}}$, HUAN-HUAN PEI ${ }^{1 *}$, JUAN SUN $^{2 *}$, QIN-XIU XIE ${ }^{1}$ and JIA-BIN LI ${ }^{1}$ \\ ${ }^{1}$ Department of Infectious Diseases, The First Affiliated Hospital of Anhui Medical University, Hefei, Anhui 230022; \\ ${ }^{2}$ Department of Science and Technology, Anhui University of Chinese Medicine, \\ Hefei, Anhui 230038, P.R. China
}

Received August 31, 2014; Accepted June 2, 2015

DOI: $10.3892 / \mathrm{ol} .2015 .3724$

\begin{abstract}
Hepatitis B virus (HBV) reactivation associated with radiotherapy is rare. The present study reports the case of a 46-year-old man that experienced fatal HBV reactivation. The patient suffered from hepatocellular carcinoma (HCC) with portal vein tumor thrombus, which was treated by radiotherapy at a daily fraction of 2 Gy over 5 weeks, up to a total radiation dose of $\sim 50 \mathrm{~Gy}$. The patient presented with fatigue, yellow sclera and abdominal distension $\sim 8$ weeks subsequent to the administration of radiotherapy. The liver function tests, including the level of total bilirubin and prothrombin time, suggested acute-on-chronic liver failure. The serum HBV-DNA level had also increased between undetectable levels and $7.2 \times 10^{4}$ copies $/ \mathrm{ml}$. Although the present patient with HCC was treated with $0.5 \mathrm{mg} /$ day entecavir for 8 weeks, in addition to radiotherapy, radiation-induced HBV reactivation occurred. The condition of the patient worsened gradually. The present study emphasizes the importance of liver function and HBV-DNA screening and pre-emptive antiviral prophylaxis prior to radiotherapy in patients with HCC.
\end{abstract}

\section{Introduction}

Hepatitis B virus (HBV) infection is a serious and common global public health problem (1). It is conservatively estimated that $>2$ billion individuals have been infected with HBV worldwide and 350 million have suffered from chronic HBV infection. In Asia and the majority of Africa, the disease is particularly prevalent and usually acquired perinatally or in childhood (1). In total, $15-40 \%$ of the infected patients will

Correspondence to: Professor Jia-Bin Li, Department of Infectious Diseases, The First Affiliated Hospital of Anhui Medical University, 218 Jixi Road, Hefei, Anhui 230022, P.R. China

E-mail: 1ijiabin948@vip.sohu.com

*Contributed equally

Key words: radiation, reactivation, hepatitis B virus, hepatocellular carcinoma develop cirrhosis, liver failure or hepatocellular carcinoma (HCC), which is currently the fifth most frequent cancer and accounts for between 300,000 and 500,000 mortalities per year (2).

Curative surgery is currently available for only $10 \%$ of patients. Due to the low tolerance of the liver for irradiation, radiotherapy is limited and is therefore not optimal for the treatment of HCC (3). Therefore, chemotherapy has become a main palliative treatment for certain inoperable patients. However, there is a considerable probability for the reactivation of $\mathrm{HBV}$ in $\mathrm{HCC}$ patients that receive chemotherapeutic drugs, which may be a fatal complication and may lead to disruption in treatment schedules (4). With the development of novel radiotherapy as 3-dimensional conformal therapy or stereotactic radiation therapy, the role of radiotherapy has become of increasing importance in the treatment of HCC, particularly for HCC patients with portal vein tumor thrombus (PVTT) or a tumor diameter $<10 \mathrm{~cm}(3-5)$.

To the best of our knowledge, previous studies have reported the occurrence of chemotherapy-associated reactivation of HBV in the literature $(6,7)$. However, the occurrence of radiotherapy-associated $\mathrm{HBV}$ reactivation is rare. Therefore, the present study reports the case of a patient that experienced fatal $\mathrm{HBV}$ reactivation during radiotherapy for $\mathrm{HCC}$ with portal vein tumor thrombosis (PVTT) formation.

\section{Case report}

A 46-year-old male patient with a long history as a HBV carrier presented with fatigue, yellow sclera and abdominal distension that had lasted for $>10$ days was referred to the First Affiliated Hospital of Anhui Medical University (Hefei, Anhui, China) on June 26, 2012. The patient possessed a family history of chronic HBV infection and a history of alcohol abuse. The patient had been diagnosed with HBV-associated HCC at the First Affiliated Hospital of Zhengzhou University (Zhengzhou, Henan, China) in April 2012.

Abdominal computed tomography with contrast medium revealed a left frontal tumor, $8.7 \mathrm{~cm}$ in diameter, in the liver with PVTT. The total liver volume of the patient was $1617.4 \mathrm{~cm}^{3}$ and the gross tumor volume was $352.7 \mathrm{~cm}^{3}$. The patient was identified to possess the following pattern of HBV markers: Hepatitis B surface antigen (HBsAg)-positive; 
anti-hepatitis B surface antibody (HBsAb)-negative; hepatitis B envelope antigen ( $\mathrm{HBe} A g)$-negative; anti hepatitis B envelope antibody ( $\mathrm{HBeAb}$ )-positive; and anti hepatitis B core antibody $(\mathrm{HBcAb})$-positive. The quantitative polymerase chain reaction (qPCR) level of HBV-DNA remained undetectable. The biochemical analyses of liver function tests revealed normal function, as follows: Alanine aminotransferase (ALT), $26 \mathrm{U} / 1$ (normal, 7-40 U/l); aspartate aminotransferase (AST), $20 \mathrm{U} / 1$ (normal, 13-35 U/l); total bilirubin (TB), $17.34 \mu \mathrm{mol} / 1$ (normal, 5-19 $\mu \mathrm{mol} / \mathrm{l}$ ); and prothrombin time (PT), $15.6 \mathrm{sec}$ (normal, 11-16 sec). The patient received prophylactic antiviral therapy with oral administration of the nucleoside analog entecavir $(0.5 \mathrm{mg} /$ day over 8 weeks $)$ in addition to undergoing radiotherapy, commencing on May 1, 2012. The patient was administered with a daily fraction of $2 \mathrm{~Gy}$, up to a total radiation dose of $\sim 50$ Gy over 5 weeks.

On June 26, 2012, 8 weeks subsequent to the first dose of radiotherapy, the patient was admitted to the Department of Infectious Diseases at the First Affiliated Hospital of Anhui Medical University due to fatigue, yellow sclera and abdominal distension that had lasted $>10$ days. The liver function tests revealed severe liver dysfunction, as follows: Albumin, $29.3 \mathrm{~g} / 1$ (normal, 40-50 g/l); ALT, $741 \mathrm{U} / 1$ (normal, 7-40 U/l); AST, $405 \mathrm{U} / 1$ (normal, 13-35 U/l); TB, $356.15 \mu \mathrm{mol} / 1$ (normal, 5-19 $\mu \mathrm{mol} / \mathrm{l}$ ); and PT, $57.5 \mathrm{sec}$ (normal, 11-16 sec). The HBV serology status continued to indicate that the patient was HBsAg-positive, HBeAb-positive, HBcAb-positive, HBsAb-negative and HBeAg-negative. However, the detectable qPCR level of HBV-DNA was $7.2 \times 10^{4}$ copies $/ \mathrm{ml}$. The laboratory characteristics for infection with the hepatitis A, C, $\mathrm{D}$ or $\mathrm{E}$ viruses were absent. As entecavir demonstrates few side-effects on liver function, the nucleoside analogue was not considered to be the cause of the changes in liver function.

On the basis of the symptoms, physical signs and laboratory examination, the patient was diagnosed with acute-on-chronic liver failure resulting from the reactivation of HBV. The patient received prompt symptomatic and supportive treatment for liver dysfunction in addition to active antiviral therapy. The duration of antiviral therapy was $\sim 8$ weeks from May 2012. However, the liver failure was further aggravated by the HBV reactivation. The breathing and circulation of the patient gradually weakened; on June 27 , the second day subsequent to admission, the patient demonstrated acute thoracodynia, dyspnea and mania. The blood gas analysis revealed a $\mathrm{PaO}_{2}$ level of $55 \mathrm{mmHg}, \mathrm{PaCO}_{2}$ level of $30 \mathrm{mmHg}$ and $\mathrm{P}_{\mathrm{A}-\mathrm{a}} \mathrm{O}_{2}$ level of $0.86 \mathrm{kPa}$. The patient succumbed to HBV reactivation at 3 weeks after the end of radiotherapy.

\section{Discussion}

HBV reactivation is a common problem that clinicians often encounter following chemotherapy or immunosuppressive therapy for disorders including hematological or solid malignancies, rheumatism and systemic lupus erythematosus. Chemotherapy-associated reactivation has been well described in the literature. Induced immunosuppression facilitates an increased viral load and antigen expression. Upon recovery of the immunity of the patient subsequent to treatment withdrawal or reduction, there is an intense immune-mediated response that eradicates HBV-infected hepatocytes and manifests clinically as asymptomatic anicteric hepatitis, severe hepatitis or even fatal liver failure (8). The aforementioned hypothesis may not apply to the pathogenesis of radiation-induced HBV reactivation due to the differences between radiotherapy and chemotherapy, and since radiotherapy usually exerts an increased effect on local tissue rather than the whole immune system.

HBV reactivation is a severe complication that occurs in patients with HCC that undergo radiotherapy, and it may occasionally be fatal. Although the present patient with HCC was treatment with entecavir in addition to radiotherapy, radiation-induced HBV reactivation occurred and led to the development of liver failure. The mortality rate directly due to HBV reactivation has been reported as $\sim 60 \%$ in previous studies (7,9-11). Therefore, awareness of this potentially life-threatening complication and regular monitoring of the clinical parameters associated with HBV reactivation during thermotherapy or radiotherapy are of considerable importance (12). To the best of our knowledge, HBV reactivation during chemotherapy is associated with baseline HBV-DNA levels and $\mathrm{HBeAg}$ positivity. Few studies have investigated the risk factors and incidence of $\mathrm{HBV}$ reactivation in patients with HBV-associated HCC undergoing radiotherapy (13). However, a recent study reports that the serum HBV-DNA level and certain dosimetric parameters, such as normal liver volume, gross tumor volume and mean radiological dose, acted as prognosis factors for HBV reactivation and should be considered carefully prior to radiotherapy (14).

The present study indicated that preemptively prophylactic administration of entecavir prior to the performance of radiotherapy may be proposed as a first-line preventive therapy in patients with HCC that are inactive carriers of $\mathrm{HBV}$, rather than as a preventive therapy once radiotherapy has commenced or as a rescue therapy when HBV reactivation has occurred. The optimal duration of entecavir administration for the prevention of radiotherapy-induced HBV reactivation has yet to be clarified. In the future, large prospective and case-controlled studies should be performed to determine the optimal duration. A previous study reported that an increased and persistent interleukin (IL)-6 density in the liver was evident following radiotherapy. IL-6 was released from irradiated endothelial cells and induce $\mathrm{HBV}$ reactivation in vitro and in vivo through the bystander effect. The signal transducer and activator of transcription 3 signaling pathway mainly plays an important role in this phenomenon reported from the literature (13). As a result, the additional studies are also required to delineate the precise mechanism on HBV reactivation subsequent to radiotherapy.

\section{References}

1. Lee WM: Hepatitis B virus infection. N Engl J Med 337: 17331745, 1997.

2. Lok AS: Chronic hepatitis B. N Engl J Med 346: 1682-1683. 2002.

3. Bruix J, Sherman M, Llovet JM, Beaugrand M, Lencioni R, Burroughs AK, Christensen E, Pagliaro L, Colombo M and Rodés J; EASL Panel of Experts on HCC: Clinical management of hepatocellular carcinoma. Conclusions of the Barcelona-2000 EASL conference. European Association for the Study of the Liver. J Hepatol 35: 421-430, 2001.

4. Jang JW, Kwon JH, You CR, Kim JD, Woo HY, Bae SH, Choi JY, Yoon SK and Chung KW: Risk of HBV reactivation according to viral status and treatment intensity in patients with hepatocellular carcinoma. Antivir Ther 16: 969-977, 2011. 
5. Kang MK, Kim MS, Kim SK, Ye GW, Lee HJ, Kim TN and Eun JR: High-dose radiotherapy with intensity-modulated radiation therapy for advanced hepatocellular carcinoma. Tumori 97: 724-731, 2011.

6. Yeo W and Johnson PJ: Diagnosis, prevention and management of hepatitis B virus reactivation during anticancer therapy. Hepatology 43: 209-220, 2006.

7. Yeo W, Lam KC, Zee B, Chan PS, Mo FK, Ho WM, Wong WL, Leung TW, Chan AT, Ma B, et al: Hepatitis B reactivation in patients with hepatocellular carcinoma undergoing systemic chemotherapy. Ann Oncol 15: 1661-1666, 2004.

8. Francisci D, Falcinelli F, Schiaroli E, Capponi M, Belfiori B, Flenghi L and Baldelli F: Management of hepatitis B virus reactivation in patients with hematological malignancies treated with chemotherapy. Infection 38: 58-61, 2010.

9. Kohrt HE, Ouyang DL and Keeffe EB: Systematic review: Lamivudine prophylaxis for chemotherapy-induced reactivation of chronic hepatitis B virus infection. Aliment Pharmacol Ther 24: 1003-1016, 2006.
10. Yeo W, Chan PK, Hui P, Ho WM, Lam KC, Kwan WH, Zhong S and Johnson PJ: Hepatitis B virus reactivation in breast cancer patients receiving cytotoxic chemotherapy: A prospective study. J Med Virol 70: 553-561, 2003.

11. Nagamatsu $H$, Kumashiro $R$, Itano $S$, Matsugaki $S$ and Sata $M$ : Investigation of associating factors in exacerbation of liver damage after chemotherapy in patients with HBV-related HCC. Hepatol Res 26: 293-301, 2003.

12. Lalazar G, Rund D and Shouval D: Screening, prevention and treatment of viral hepatitis B reactivation in patients with haematological malignancies. Br J Haematol 136: 699-712, 2007.

13. Chou CH, Chen PJ, Jeng YM, Cheng AL, Huang LR and Cheng JC: Synergistic effect of radiation and interleukin-6 on hepatitis B virus reactivation in liver through stat 3 signaling pathway. Int J Radiation Oncology Biol Phys 75: 1545-1552, 2009.

14. Huang W, Zhang W, Fan M, Lu Y, Zhang J, Li H and Li B Risk factors for hepatitis B virus reactivation after conformal radiotherapy in patients with hepatocellular carcinoma. Cancer Sci 105: 697-703, 2014. 